\title{
El idioma mexicano visto desde las escuelas de pueblos tlaxcaltecas, 1900-1930
}

\author{
The Mexican Language Seen from \\ the Schools of Tlaxcaltecas Towns, 1900-1930
}

\author{
Daniel Gibran Castillo Molina \\ Centro de Investigación y de Estudios Avanzados \\ del Instituto Politécnico Nacional, Unidad Monterrey \\ simpatiaazul@hotmail.com \\ daniel.castillom@cinvestav.mx
}

\begin{abstract}
Resumen
En este artículol analizo el uso oral de la lengua náhuatl en el contexto educativo de pueblos pertenecientes a la cabecera municipal de Santa Ana Chiautempan, Tlaxcala, antes y después de la Revolución mexicana. Para ello, rescato a los docentes originarios de pueblos sujetos y de la misma cabecera. El objetivo principal es mostrar el uso que se hace de una lengua indígena en los primeros años educativos de niños hablantes para que éstos puedan comprender sus clases y su acercamiento al español. Muestro que los maestros hablantes de la lengua nativa local de hecho la utilizaban en las comunidades y las aulas, aun cuando su uso fue tema de fuertes controversias entre los pedagogos mexicanos durante las primeras décadas del siglo XX, tanto antes como después de la fundación de la Secretaría de Educación Pública en 1921
\end{abstract}

Palabras clave: lengua náhuatl, Tlaxcala, educación rural, maestros, castellanización.

\begin{abstract}
This article analyses the oral use of the nahuatl language, in the context of formal education, in the indigenous towns belonging to the municipality of Santa Ana Chiautempan, Tlaxcala, before and after the Mexican Revolution. I follow the trajectories of teachers who were from both the municipal headtown and surrounding pueblos. The main purpose is to show that they did use the indigenous

${ }^{1}$ Este artículo es resultado de mi tesis de maestría para obtener el grado de maestro en Ciencias en la especialidad en Investigaciones Educativas del Departamento de Investigaciones Educativas CINVESTAV, sede sur Ciudad de México, a principios del 2017.
\end{abstract}


language with native, monolingual children during their first years of schooling, in order to facilitate their comprehension and further acquisition of Spanish. I show that many teachers who were bilingual did in fact use the local language to enable children both to understand and acquire further knowledge of Spanish, even though this practice was a topic of strong controversy among Mexican pedagogues during the first two decades of the twentieth century and after the founding of the federal Ministry of Public Education (SEP) in 1921.

Keywords: Nahuatl language, tlaxcala, rural education, teachers, Spanish language.

\section{Introducción}

Abordar la historia del uso de una lengua indígena en el devenir educativo mexicano ha sido un reto para los diferentes investigadores interesados en dicha temática. Desde el siglo XVI la Corona española impulsó la castellanización de los nativos en el continente americano. Bárbara Cifuentes señala que se consideraba al español una lengua superior necesaria para civilizar a los nativos (Cifuentes, 2002: 47). A pesar de lo anterior, las lenguas indígenas con frecuencia fueron utilizadas por los religiosos para el adoctrinamiento cristiano y la educación. En 1696 Carlos II estableció el español como único idioma oficial válido en los aspectos gubernamentales (Cifuentes, 1998). En el siglo xix el discurso de la igualdad de ciudadanía en el México independiente impidió que se debatiera la diversidad poblacional y lingüística del país, por lo que el tema de las distintas lenguas se fue relegando de los ámbitos políticos y educativos. Los habitantes de muchos pueblos nunca dejaron de hablar su lengua materna, ya sea de forma monolingüe o bilingüe; era común su empleo oral y formaba parte de su cotidianidad. Lo anterior ocasionó que, en la mayoría de los casos, la práctica de una lengua distinta de la dominante no fuera expresada por las personas de forma escrita, favoreciendo la oralidad.

Según Alicia Martín y Carlos Escalante, los trabajos analíticos sobre la historia de la educación indígena corresponden a procesos educativos en la primera mitad del siglo xx y en la época colonial (Martín y Escalante, 2017: 349). Algunos de los estudios que han abordado el uso de las lenguas en la historia de la educación mexicana son los siguientes: Bárbara Cifuentes (1998, 2002) analizó la política lingüística de México, otorgándole atención al rubro educativo durante los siglos XIX y XX; Shirley Brice Heath (1970) describió la política lingüística a través de toda la historia de México retomando a educación; Ariadna Acevedo (2011a, 2011b y 2012), a través de sus investigaciones, ha rescatado algunas evidencias del uso del totonaco y el náhuatl por parte de algunos profesores en las escuelas de la Sierra Norte de Puebla a finales del siglo XIX y principios del XX. 
Respecto al caso tlaxcalteca, Jacqueline Messing (2003 y 2006) abordó para el municipio de Contla la historia oral de sus habitantes y el impacto del uso del mexicano en espacios como la escuela; Elsie Rockwell (1994, 2004, 2006a, 2006b, 2007a y 2007b), por su parte, ha expuesto la importancia del mexicano en la educación de los niños de la zona de La Malintzi a través de su historia educativa. Sin embargo, es necesario incorporar nuevos estudios no sólo para Tlaxcala sino para otros estados, que sin duda contribuirán a la historiografía educativa indígena en México.

Este estudio se centró en un corpus limitado debido a la poca información escrita que existe: algunos documentos en los fondos histórico y educativo del Archivo Histórico del Estado de Tlaxcala (AHET) ofrecieron fojas muy explícitas del uso del mexicano por parte de los docentes para la educación y castellanización de los niños. Una mención del profesor Andrés Lima y su bilingüismo, el informe escolar de un director sobre el uso del mexicano por parte de su ayudante Ricarda Muñoz en el primer grado y las peticiones de los mismos padres de familia de contar con profesores hablantes de la lengua materna de la población, son ejemplos claros de la importancia y uso de una lengua indígena en la educación de estos pueblos y barrios de Santa Ana Chiautempan. Los principales objetivos son demostrar que los profesores utilizaron la lengua materna en la educación de los niños de estos pueblos, y el posicionamiento de los padres de familia locales frente al uso del idioma mexicano.

Como sustento teórico y metodológico recupero el análisis de la microhistoria mexicana de Luis González y González (1997a y 1997b). Carlos Escalante Fernández plantea que para la historia de la educación los espacios municipales pueden rescatar la vida de los profesores: sus comportamientos, su relevancia local y su aceptación vecinal (Escalante, 2006: 84). El mismo Escalante sustenta que a través de la mirada local se puede ganar tiempo y profundidad de estudio en temas educativos (Escalante, 2006: 82). Así, analizo a los profesores y padres de familia locales con mayor detenimiento dentro de su microespacio para conocer lo que realmente sucedía en esas escuelas, sacando a la luz el uso de una lengua ajena a la dominante en la educación, proponiendo mirar de una manera distinta la historia de la educación mexicana y rescatar lo que se ignoraba o les causaba polémica a los grandes pensadores y pedagogos mexicanos de la época, que no debatían la utilidad, a principios del siglo Xx ni en los primeros años de la educación posrevolucionaria, de las lenguas indígenas en la educación de los pueblos hablantes.

En este artículo presento un panorama general sobre la situación de las distintas lenguas mexicanas en el contexto educativo desde el siglo XIX hasta los primeros años del siglo XX. Posteriormente, muestro el contexto de los pueblos pertenecientes al municipio de Santa Ana Chiautempan durante la temporalidad de estudio. Argumentaré con ejemplos el uso del mexicano, por parte de algunos profesores, como auxiliar en clases con niños monolingües 
durante la temporalidad de estudio. Finalmente, dedico un apartado a un curso preparatorio en el que participaron algunos profesores para la castellanización de los niños.

\section{La educación mexicana y las lenguas}

Durante la evangelización de los nativos en los nuevos territorios conquistados por la Corona española las órdenes religiosas utilizaron en buena medida las lenguas indígenas de los pueblos con el propósito de llevar a cabo una cristianización más eficiente.

En el México independiente el discurso de la igualdad de ciudadanía obligó a considerar a toda la población sin distinción de castas, dejando de lado una educación planeada para la población indígena. Dotarlos de una visibilidad durante el siglo XIX es difícil: en el plano político oficial dejaron de existir los pueblos de indios, descuidando el tema de las lenguas, la identidad y la cultura de los distintos pueblos de México.

En 1820 el liberal Juan Rodríguez Puebla buscó un proyecto educativo planeado para los indígenas en el que se emplearían sus lenguas para la enseñanza (Castillo, 2017: 75). Sin embargo, políticos liberales como José María Luis Mora no avalaron una educación especializada y mucho menos la utilización de las lenguas nativas. Shirley Brice Heath señala que los portavoces de los indios eran pocos como para alterar la orientación de la política educativa durante el primer siglo de independencia (Heath, 1970: 101-102). Durante el siglo XIX ni existió ni se debatió una política lingüística unificada en México; el único idioma oficial válido era el español. La educación impartida en el México independiente era asimiladora e integracionista, con el objetivo de favorecer la construcción de una nación homogénea y mestiza. Lo anterior, no impidió que algunos obispados continuaran con la tarea de educar a los indígenas bajo sus propias lenguas. Cifuentes (2002: 65) señala que para educarlos no era necesario anteponer la enseñanza del español. La Iglesia tenía la tradición de imprimir textos y gramáticas en lenguas indígenas, sobre todo en náhuatl.

En 1857 el liberal Ignacio Ramírez instó a que se publicaran materiales educativos en lenguas indígenas (Castillo, 2017: 77). Otros como Francisco Pimentel no discutieron la utilidad de educar a los indígenas. Martín y Escalante (2017: 349) señalan que hace falta saber más sobre la educación de los indígenas en el siglo XIX. Durante estos años se formaron la Sociedad Mexicana de Geografía y Estadística y la Academia Mexicana de la Lengua, despertando el interés por las distintas lenguas de México, aunque sus miembros ignoraron la heterogeneidad lingüística del país, sus estudios comenzaron a debatir el tema lingüístico en México.

A principios del siglo xx en el plano político y social no se debatía la diversidad lingüística en México. Al parecer, no existía ninguna legislación o reglamento sobre el uso de las 
lenguas vernáculas en la educación. Los lingüistas como Francisco Belmar reconocieron la diversidad idiomática de México; Heath señala que la propuesta educativa de la Sociedad Indianista era que se produjeran textos en lenguas vernáculas, sobre todo para cuestiones de enseñanza agrícola, pero ni funcionarios ni políticos hicieron caso de estas propuestas (1970: 122-124).

En el plano cotidiano de los pueblos se vivía de una forma distinta. Eric Hobsbawm plantea que, a pesar de la tendencia por lograr la unificación de las naciones mediante una lengua nacional, los distintos pueblos de cada nación mantenían en cierta medida sus propias lenguas, a pesar de que patrocinaban el uso de la lengua dominante (Hobsbawm [1991], citado en Castillo, 2017: 77). En Tlaxcala era común que los pueblos siguieran usando su lengua materna, aunado al uso del español; Hilario Vázquez Santa Anna relata que los pobladores de las faldas de La Malintzi usaban su lengua dentro del pueblo (1928: 12). Manuel Urquía describe el uso del mexicano al señalar que se hablaba con mucha facilidad y frecuencia, particularmente en las discusiones de los habitantes y sus relaciones familiares (1942: 16). Para la época, el bilingüismo era algo usual en Tlaxcala, pues muchos pueblos conocían y usaban el español y eso no suponía dejar de lado su lengua materna (Castillo, 2017: 80).

El 18 de junio de 1911 se puso sobre la mesa el tema de las lenguas indígenas en la educación mexicana. Durante el gobierno provisional de León de la Barra se autorizó la instrucción rudimentaria que tenía por objeto "enseñar al individuo de raza indígena, a hablar, leer y escribir en castellano" (Aguirre [1973], citado en Castillo, 2017: 78). Gregorio Torres Quintero rediseñó las rudimentarias, patrocinando el método directo que consistía en la enseñanza del español sin la utilización de las lenguas indígenas. Los criterios sobre la educación de los indígenas variaban y no existió acuerdo de cómo lograrlo, aunque ya existía la preocupación. Hacia 1921 se desconocía realmente el número de lenguas y hablantes. Heath sostiene que durante la etapa de José Vasconcelos no se admitía que las lenguas vernáculas fueran un instrumento educativo (Heath, 1970: 137). Pedagogos como Rafael Ramírez retomaron el método directo de Quintero en la castellanización impulsada por la fundada Secretaría de Educación Pública (SEP). Moisés Sáenz comenzó a considerar el uso de las lenguas como auxiliares para los profesores.

En Tlaxcala no existía ninguna ley o reglamento educativo que señalara o prohibiera el uso del mexicano en las escuelas, no hay indicios de que antes o durante los primeros años posrevolucionarios existieran quejas o preocupaciones por parte de los políticos estatales del uso del mexicano en la educación tlaxcalteca. Los profesores del sistema estatal que hablaban mexicano utilizaban la lengua sin que nadie se los señalara. Este trabajo demostrará que los mismos pueblos pedían un profesor hablante de mexicano y español, y que era fundamental contar con el conocimiento de la lengua materna de los pueblos para poder educar a los niños. 


\section{Panorama social de los pueblos y barrios de Chiautempan}

El municipio de Chiautempan se encuentra en la zona centro sur del estado de Tlaxcala, a escasos kilómetros de la ciudad de Tlaxcala. Entre 1900 y 1930 sus pueblos como Tetlanohcan y Tlaltelulco² se extendían hacia las faldas del volcán La Malintzi conocido localmente como Matlalcueyétl.

La zona centro sur contaba con la mayor densidad de población, ${ }^{3}$ y buena parte de la mano de obra provenía de los pueblos asentados en las faldas de La Malintzi. Chiautempan contaba con pueblos reconocidos como entidades políticas y con su propia delimitación territorial. La lengua náhuatl, conocida en estos pueblos como mexicano, era su lengua materna; además de ser pueblos obreros se dedicaban al comercio de madera y carbón, y a la fabricación de trementina, que los habitantes vendían en la cabecera y otros municipios aledaños. ${ }^{4}$ En los barrios de Ixcotla y Texcacoac, más cercanos a la cabecera, los informes escolares detallan que los habitantes eran tejedores de lana. ${ }^{5}$

El grado de bilingüismo también variaba entre los pueblos, pues en cierta medida dependía de su cercanía con la cabecera y los diferentes oficios que desempeñaban los habitantes (Castillo, 2017: 82). Debido a la constante interacción social y comercial, los habitantes contaban con cierto uso del español como segunda lengua. Dolores Plá señala que en el censo de 1921 a la población indígena por primera vez se le clasificó como tal por el criterio lingüístico (Plá, 2005: 67). No es fácil determinar qué tanto usaban y conocían el español las personas ni el grado de conocimiento de una lengua indígena, por lo que las cifras de los censos no son confiables.

${ }^{2}$ El nombre de los pueblos y barrios que componían Chiautempan durante la temporalidad de estudio son los siguientes: La Magdalena Tlaltelulco, San Bartolomé Cuahuixmatlac, San Francisco Tetlanohcan, San Pedro Tlalcuapan, San Pedro Xochiteotla, barrio de San Rafael Tepatlaxco, barrio de Texcacoac, barrio de Guadalupe Ixcotla (barrio grande, contaba con el barrio adyacente de Santa Cruz Guadalupe Ixcotla), barrio de Melendeztla y barrio de Muñoztla (ambos formaban parte de Tlalcuapan). A partir de 1994 son municipios de nueva creación Tetlanohcan y Tlaltelulco.

${ }^{3}$ Datos del censo de 1900 a cargo del doctor Antonio Peñafiel. División Territorial de la República Mexicana, Estado de Tlaxcala. Datos de la Secretaría de Agricultura y Fomento, Dirección de Estadística. Tercer Censo de Población, capítulo III Edades por entidades federativas, distritos y municipalidades y Datos de la Dirección General de Estadística, Quinto Censo de Población 1930. Toda la información se ubica en el Instituto de Geografía de la Universidad Nacional Autónoma de México (UNAM).

${ }^{4}$ Los informes de algunos profesores indican que los padres de familia eran carboneros y leñadores. Archivo Histórico del Estado de Tlaxcala (AHET), Fondo: Histórico, Sección: Secretaría de Educación Pública (SEP), Año: 1926, Caja: 142, Exp.: 32, f. 1; AHET, Fondo: Histórico, Sección: SEP, Año: 1928, Caja: 197, Exp.: 24, f. 13; AHET, Fondo: Histórico, Sección: SEP, Año: 1928, Caja: 211, Exp.: 11, f. 11, Chiautempan, Tlaxcala.

${ }^{5}$ AHET, Fondo: Histórico, Sección: SEP, Año: 1929, Caja: 251, f. 8; AHET, Fondo: Histórico, Sección: SEP, Año: 1930, Caja: 277, f. 13, Chiautempan, Tlaxcala. 


\section{Panorama educativo de los pueblos y barrios de Chiautempan}

De 1900 a 1930 prácticamente todas las escuelas de estas localidades se ubicaban en casas particulares de los vecinos del lugar. Contaban con la tradición de tener escuelas separadas tanto de niños como de niñas, aunque compartían el mismo espacio para la instrucción, muchas veces en cuartos separados por una cortina. ${ }^{6}$

Los informes de agentes municipales y profesores proporcionan datos sobre la existencia de escuelas en estos pueblos antes de 1900. El profesor Andrés Angulo relató en un informe que en Tepatlaxco había ya una escuela desde 1890.? En 1922 el informe del agente municipal de Tlalcuapan, Pedro Muñoz, señalaba que desde 1890 ya había una escuela mixta en esa población. ${ }^{8}$

Las escuelas de estos pueblos y barrios fueron unidocentes, separadas por género, que a lo largo de los años veinte poco a poco se fueron fusionando en unidocentes mixtas (Castillo, 2017: 40). Algunos directores contaban con ayudantes, como en las escuelas de Ixcotla y Tlalcuapan, en 1928. Las escuelas contaban con dos años o grados escolares para las niñas y hasta tercer grado para los niños. El barrio de Ixcotla, en 1926, ya contaba con tres escuelas: niños, niñas y Santa Cruz Guadalupe.

De 1900 a 1917 las escuelas tenían el nombre de escuelas elementales y las había para niñas, niños o mixtas; en 1917 su denominación fue modificada, aparte de lo elemental se les agregó un número de identificación escolar. El término "rural" se popularizaría en el estado de Tlaxcala después de 1920, salvo en las escuelas de las "rancherías" y en las rudimentarias. En el año de 1926 la federalización ingresó al municipio de estudio, se creó una escuela rural federal en el barrio de Melendeztla; el inspector federal Fernando Ximello mencionó detalles que no habían sido mencionados en los informes de inspectores estatales como la "raza" y la lengua de los niños del lugar. Ximello, en su visita del 28 de septiembre de 1926, se refirió al tipo de "raza" de los niños en la escuela, clasificándolos como indígenas que "no hablan bien el castellano. ${ }^{9}$ En 1928 se federalizó la escuela de Xochiteotla, con lo que se estableció definitivamente ese sistema educativo. El inspector federal Salomón Pérez señalaba en uno

\footnotetext{
${ }^{6}$ AHET, Fondo: Revolución y Álvaro Obregón (FRRO), Sección: Instrucción Pública (IP), Año: 1902, Caja: 20, Exp: 23, f. 28; AHET, Fondo: FRro, Sección: IP, Año: 1917, Caja: 346, f. 34, Tlaxcala, Tlaxcala.

${ }^{7}$ Informe del profesor Andrés Ángulo al celador de instrucción pública. AHET, Fondo: FRRO, Sección: IP, Año: 1900, Caja: 20, f. 23, Chiautempan, Tlaxcala.

${ }^{8}$ Informe del agente municipal Pedro Muñoz al director de educación. AHET, Fondo: Histórico, Sección: SEP, Año: 1922, Caja: 73, f. 2, Chiautempan, Tlaxcala.

${ }^{9}$ Expediente de la escuela rural federal de Melendeztla, Chiautempan, Tlaxcala. Archivo General de la Nación (AGN), Archivo Histórico de la Secretaría de Educación Pública (AHSEP), Fondo: Dirección General de Educación Para Estados y Territorios (DGEPYT), Año: 1926, Caja: 4037B, Referencia: IV/161(IV-14) /21187, Ciudad de México.
} 
de sus informes que los niños eran "indígenas tlaxcaltecas" que hablaban castellano.. ${ }^{10}$ En 1927 el propio inspector Ximello afirmaba que los niños en la escuela rural federal de Texcacoac eran mestizos y hablaban castellano. ${ }^{11}$ Los datos anteriores nos indican que efectivamente en estos pueblos había niños que hablaban el mexicano y español.

\section{La lengua que sí era hablada en la escuela: el mexicano}

Un vacío que prevalece en la historiografía educativa mexicana es la cuestión del rescate y la importancia que pudieron tener las lenguas vernáculas en la educación de los pueblos indígenas. Aunque se pretendió educar a los indígenas ignorando sus idiomas y anteponiendo el español como único idioma válido, el uso de las lenguas como auxiliar en las clases siempre fue recurrente tanto para la Iglesia como para el sistema educativo laico.

En el siglo xIx el náhuatl ya no era admitido como una lengua para el dominio público; su uso se relegó a ámbitos locales y casi siempre de forma oral. Elsie Rockwell plantea que después de las Leyes de Reforma los protestantes, particularmente los metodistas, compitieron con la Iglesia católica en la difusión de la cultura escrita y alentaron el estudio de las sagradas escrituras en español (Rockwell, 2006b: 53). En Tlaxcala, el mexicano seguía siendo el idioma de sus habitantes más concretamente de forma oral. Durante el porfiriato ${ }^{12}$ el gobernador de Tlaxcala, Próspero Cahuantzi, hablaba mexicano con los caciques de los pueblos en la plaza pública de la capital tlaxcalteca.

En las escuelas de los pueblos tlaxcaltecas hablantes de mexicano existió una convivencia entre este idioma y el español. Los pueblos, para ser reconocidos administrativa y políticamente como tales, preparaban a personas letradas en español para cubrir los cargos civiles que se requerían, sin embargo, el uso oral de la lengua materna seguía prevaleciendo. La naturalidad del mexicano en Tlaxcala y la falta de información en los documentos oficiales sobre la lengua y la población hablante hacen que sea un reto descubrir dicha temática. Este artículo intenta exponer algunos ejemplos de cómo el uso del mexicano era utilizado de forma cotidiana en la vida escolar junto con el español.

${ }^{10}$ Informe del inspector federal Salomón Pérez. AGN, AHSEP, Fondo: DGEPYT, Año: 1928, Caja: 40373, Referencia: IV/161 (IV-14) /21187, Ciudad de México.

${ }^{11}$ Informe del inspector federal Fernando Ximello. AGN, AHSEP, Fondo: DGEPYT, Año: 1927-1965, Caja: 8668/5, Referencia: IV/161 (IV-14) /21289, Ciudad de México.

12 Periodo mexicano que comprende la presidencia de Porfirio Díaz (1876-1910). 


\section{Los maestros hablantes de mexicano: su uso en la escuela}

Rockwell señala que en la región de La Malintzi "fue posible que algunos maestros usaran la lengua náhuatl por lo menos oralmente" (Rockwell, 2004: 332). Los profesores de los pueblos a los que me refiero en este trabajo formaban parte del sistema educativo estatal y eran originarios ya fuera de la cabecera de Chiautempan o de las localidades pertenecientes a la misma durante la temporalidad de estudio. ${ }^{13}$ Dado lo anterior, es un hecho que algunos de estos profesores conocían y hablaban el mexicano, incluso que para ellos fuera su primera o segunda lengua y que la usaron en su vida escolar diaria para la enseñanza y comunicación con los padres de familia de estos pueblos (Castillo, 2017: 88). Coronado señala que siempre ha existido el empleo de una lengua indígena por parte de los maestros como apoyo a la instrucción y a la castellanización (Coronado, 1999: 51).

En estos pueblos ambas lenguas se usaban en la vida cotidiana; por ejemplo, el español era usado en el comercio y el mexicano en las relaciones familiares. El profesor Claudio Hernández, entrevistado por Rockwell y Oresta López en los años noventa, fue profesor en Tetlanohcan a partir de 1940. Él señala que todos los pueblos de las faldas de La Malintzi hablaban el náhuatl como primera lengua (Rockwell y López, 1992: 9). Macrina Cuapio afirma que en Tetlanohcan los habitantes eran hablantes de náhuatl, y que hubo un profesor durante "muchos años" que hablaba náhuatl en la escuela, pero no indica la fecha ni el nombre de éste (Cuapio, 2010: 17).

Considero que los profesores con conocimientos de mexicano usaban la lengua de forma natural y cotidiana, asunto que pasó inadvertido para las autoridades educativas estatales, así como para el celador de instrucción pública de principios del siglo XX, Luis Langle, y el posterior inspector educativo, Santos Galicia, encargados de la zona de Chiautempan, quienes en ninguno de sus informes hicieron alusión alguna al origen de la lengua materna de estas poblaciones ni al uso del mexicano en la educación de los niños. El mexicano tampoco era la lengua exclusiva de los profesores para la enseñanza, más bien funcionaba como un auxiliar en las clases, sobre todo con niños monolingües del primer grado.

Dada la naturaleza del tema fue un reto descubrir documentos que trataran el uso del mexicano en las escuelas de estos pueblos; más bien me encontré con algunas quejas y peticiones de los mismos padres de familia por contar con profesores hablantes de la lengua materna de los niños. Los casos que a continuación presentaré comprueban el uso de una lengua vernácula para la educación de los niños, además del empleo del español. En un expediente que tenían que llenar los profesores por mandato de la dirección educativa

\footnotetext{
${ }^{13}$ En mi tesis de maestría demostré, con base en una diversidad de documentos, los orígenes y la vida magisterial de la mayoría de los profesores que dieron clases en mi espacio de estudio, entre 1900 y 1930 (Castillo, 2017).
} 
tlaxcalteca, se les preguntaba si impartían moralidad y cívica a los alumnos. En 1919 el profesor Andrés Lima ${ }^{14}$ contestó que sí había impartido esas materias a sus alumnos y expresa lo siguiente: "Y como me encuentro en un lugar donde hablan el mexicano me veo obligado, para hacerme entender de hablar en su idioma". ${ }^{15}$ El caso anterior ejemplifica que Lima usaba dicha lengua para impartir sus clases. Lima provenía de una familia acomodada en la que había varios profesores. Durante la investigación no se pudo precisar si el mexicano era su primera o su segunda lengua.

En 1922, a través del agente municipal Pedro Muñoz y del mismo gobernador tlaxcalteca en turno, Rafael Apango, los padres de familia del pueblo de Tlalcuapan por haberles enseñado "las letras del alfabeto castellano a sus hijos", le expresaron al profesor Petronilo Tzontlimatzi lo siguiente:

[...] manifiesta el pueblo digo no queremos que nuestros hijos que sean más sabios sino que siquiera sepan que los enseño el profesor saber (contar y escribir) es lo único que pide el pueblo; porque en los años pasados han venido profesores en este pueblo pero emos [sic] visto que vuestros hijos no ha adquirido nada de conocimientos, pero cuando vino enuestro pueblo el señor profesor Petronilo Tzontlimatzi fue que les dio las primeras letras del alfabeto castellano a vuestros hijos i quedamos los del pueblo muy agradecidos $[. . .]^{16}$

El uso del mexicano en clase por parte de Tzontlimatzi ${ }^{17}$ es algo que no puedo comprobar fehacientemente, no encontré documento alusivo al tema. Tlalcuapan, su pueblo natal, tenía como primera lengua el mexicano. Los niños de estos poblados aprendían o comenzaban a familiarizarse con el español justo en las escuelas. Rockwell menciona que, a pesar del desplazamiento de la lengua vernácula, se "usaba la lengua local para comunicarse con los padres y los niños monolingües, aunque en clase insistían en el uso del español" (Rockwell, 2006b: 205). Hasta entrada la década de los setenta, Jacqueline Messing argumenta que en los pueblos de La Malintzi usaban el mexicano como auxiliar en clase, pero los padres pedían que sus hijos aprendieran español (Messing, 2003: 93).

\footnotetext{
${ }^{14}$ Andrés Lima nació en el año de 1872 en el municipio de Chiautempan, contaba con el cuarto grado elemental de estudios, en 1919 llevaba 22 años de experiencia docente.

${ }^{15}$ Expediente de Andrés Lima. AHET, Fondo: FRRO, Sección: IP, Año: 1919, Caja: 27, Exp: 58, f. 3, Chiautempan, Tlaxcala.

${ }^{16}$ Carta del agente municipal Pedro Muñoz del barrio de Muñoztla, Tlalcuapan al gobernador en turno. AHET, Fondo: Histórico, Sección: SEP, Año: 1922, Caja: 73, Exp: 14, f. 2, Chiautempan, Tlaxcala (se respeta la ortografía original del documento).

${ }^{17}$ Petronilo Tzontlimatzi nació en el pueblo de Tlalcuapan en el año de 1881, en 1919 llevaba ya 13 años de experiencia docente.
} 
La enseñanza de las materias probablemente se daba mayormente en español, pero eso no significa que se dejara de lado la lengua local en el aula; existía una especie de simbiosis entre el mexicano y español, de esa forma los niños más pequeños podían comprender sus lecciones. En 1928 el director de la escuela mixta de Tlalcuapan, Guadalupe Bello, originario del mismo pueblo, describía las labores desarrolladas por sus dos ayudantes, Ricarda Muñoz e Isabel Méndez. Bello señala explícitamente que Muñoz utilizaba el mexicano para que los niños comprendieran sus clases diarias: "La señorita Ricarda Muñoz sobresalió en sus trabajos de enseñanza en lenguaje porque sabe hablar el mexicano, les iba traduciendo al castellano las palabras y sólo así consiguió algún adelanto con sus alumnos de ambos sexos". ${ }^{18}$ El ejemplo anterior nos indica claramente que Muñoz hablaba mexicano y usaba dicha lengua para dar las clases. Muñoz era originaria de la cabecera y lo más importante es que ella precisamente impartía el primer grado, justo cuando los niños más pequeños empezaban a ir a la escuela. Considero que el uso de la lengua indígena prevalecía en los pueblos y fue clave para la castellanización de los niños, en contradicción con lo propuesto a nivel federal por algunos pedagogos destacados, es decir, "el método directo". Inclusive, en el caso anterior, Bello parece pensar que sólo gracias a la traducción que hacía Muñoz era posible conseguir "algún adelanto". Los niños hablantes del mexicano, muchos monolingües, dependían del apoyo del profesor para empezar a comprender sus clases.

La enseñanza del español podía verse reflejada en el segundo grado. Bello indica posteriormente que "la señorita Isabel Méndez, aunque sin saber el mexicano, pero como sus alumnos y alumnas son de segundo año, ya le entendían mejor y obtuvo buenos resultados en su enseñanza". ${ }^{19}$ Aunque Méndez no sabía mexicano pudo impartir sus clases porque Muñoz, mediante el uso de la lengua materna, ya les había enseñado el español a los niños de primer grado. ¿De qué forma enseñaban español?, ¿cuáles eran sus métodos? Es muy difícil plantear de qué forma enseñaban, puesto que nunca encontré ninguna información relativa al tema. Bello nos detalla que Muñoz "traducía al castellano las palabras y sólo así consiguió algún adelanto". En 1940, en Tetlanohcan, Rockwell y López le preguntaron al profesor Hernández si usaba el náhuatl de forma cotidiana en sus clases, a lo que éste contestó que normalmente sí lo hacía. Relató que se convertía en maestro bilingüe: "Y sí me entendían, Y sí podíamos ... este... ya después hacer un poquito más a un lado el náhuatl para convertir, es decir, para hacer hablar más español" (Rockwell y López, 1992: 11). El mismo Hernández detalla que enseñaba español a sus alumnos utilizando el mexicano. Sostengo la

\footnotetext{
${ }^{18}$ Informe del director Guadalupe Bello a la Secretaría de Educación de Tlaxcala (las cursivas son mías). AHET, Fondo: Histórico, Sección: SEP, Año: 1928, Caja: 100, Exp: 2, f. 24, Chiautempan, Tlaxcala.

${ }^{19}$ Informe del director Guadalupe Bello a la Secretaría de Educación de Tlaxcala. AHET, Fondo: Histórico, Sección: SEP, Año: 1928, Caja: 100, Exp: 2, f. 24, Chiautempan, Tlaxcala.
} 
idea de que a principios del siglo xx en estos poblados existían más niños monolingües de mexicano, por lo que era muy necesario conocer su lengua materna.

\section{El curso preparatorio}

El uso de un curso preparatorio era algo que se menciona en un oficio de $1917 .^{20}$ Sin embargo, no se hace alusión alguna sobre sus fines. Rockwell plantea que se usaba para "aprender hablar español si no lo hablaban" (Rockwell, 2006b: 172). Este trabajo precisamente corroborará que una de las finalidades de ese curso era la castellanización de los niños. Personajes como Andrés Lima en Ixcotla, Teresa Méndez en el pueblo de Cuahuixmatlac y Guadalupe Bello en Tetlanohcan, empleaban dos "secciones" en primer grado: "A" y "B". En estas secciones se familiarizaba al alumno con el español como lo hizo Ricarda Muñoz para el caso que ya hemos citado. Lima reportaba en 1930 que "aprendieron a hablar en castellano 3 niños y 5 niñas" en la escuela mixta de Ixcotla en primer grado, que contaba de 35 alumnos en el grupo "A" y 55 en el grupo "B". ${ }^{21}$ El informe de Teresa Méndez detalla que "veinticinco niñas aprendieron a hablar el castellano", esto en su primer grado en la escuela de niñas de Cuahuixmatlac que contaba con 33 alumnas en primer grado. Lo anterior indica que casi todas hablaban sólo su lengua materna cuando ingresaron a la escuela. ${ }^{22}$ La información sobre Méndez es escasa. Era originaria de San Pablo Apetatitlán, municipio cercano a Chiautempan, donde también era común el idioma mexicano, por tanto, sostengo como hipótesis que sabía la lengua. Otra profesora originaria de Apetatitlán fue María Luisa Alducín quien menciona que 32 niñas de 34 asistentes "aprendieron castellano" en su primer grado en Tetlanohcan. ${ }^{23}$

No existe diario o informe que indique cómo enseñaban español; sostengo, hipotéticamente, que usaban la oralidad como mayor recurso. Hill y Hill demostraron que, ya en los años setenta en esos pueblos, a menudo el primer acercamiento con el español era mediante juegos y discusiones orales en la escuela (Hill y Hill, 1999: 133).

\section{Los no "hablantes" de mexicano y los padres de familia}

Considero que un profesor identificado con las poblaciones y hablante de mexicano era fundamental para la comunicación diaria. Un profesor falto de sensibilidad y desconocedor

${ }^{20}$ Oficio relativo a los cursos preparatorios. AHET, Fondo: FRRo, Sección: IP, Año: 1917, Caja: 26, Exp: 2, f. 24, Tlaxcala, Tlaxcala.

${ }^{21}$ Informe de Lima a la Dirección Estatal de Educación. AHET, Fondo: Histórico, Sección: SEP, Año: 1930, Caja: 277, Exp: 13, f. 50, Chiautempan, Tlaxcala.

22 Informe de Méndez a la dirección estatal de educación. AHET, Fondo: Histórico, Sección: SEP, Año: 1930, Caja: 277, Exp: 18, Fs: 50, Chiautempan, Tlaxcala.

${ }^{23}$ Informe de Alducín a la dirección estatal de educación. AHET, Fondo: Histórico, Sección: SEP, Año: 1930, Caja: 270, Exp: 20, Fs: 25, Chiautempan, Tlaxcala. 
del lenguaje nativo, podría no sólo tener problemas de comunicación sino, incluso del desarrollo de sus clases (Castillo, 2017: 96).

El inspector pedagógico Wenceslao Téllez mencionaba, en 1921, que la directora de la escuela de niñas de Tetlanohcan, Felicitas Ugalde, no tenía resultados satisfactorios en sus clases. Ugalde no era hablante de mexicano, por tanto, las niñas no tenían resultados satisfactorios: "De la entrevista obtenida con las autoridades y algunos vecinos del pueblo, informo que realmente unos y otros están descontentos con la actual directora, porque el tiempo que tiene de prestar sus servicios no se han notado resultados satisfactorios de su trabajo, y que desean sea nombrada la señora Isabel Pérez, que a su juicio es competente y sabe el idioma mexicano, para hacerse comprender con los niños" ${ }^{24}$

El ejemplo anterior nos sugiere que los pobladores preferían a la profesora Isabel Pérez, que hablaba mexicano, para impartir las clases. Posteriormente, Ugalde fue destituida del puesto y Pérez tomó la escuela de niñas del poblado. En 1926, en el mismo Tetlanohcan, el agente municipal Manuel Rugerio hacía mención en una solicitud de que el profesor Evaristo Rodríguez, originario del lugar, había tenido avances con sus alumnos, y solicitaba al director de Educación, Aguillón de los Ríos, que no fuera destituido del lugar porque "como cualquier otro que viniera, tropezaría con las dificultades de la distancia, del desconocimiento del idioma y de la confianza del pueblo". ${ }^{25}$ Las palabras del agente indicaban que el uso de la lengua materna era importante para los primeros años de educación de los niños y que un profesor hablante iba a contar con "mayor confianza" del mismo pueblo que uno no hablante.

En 1922 la profesora Josefina Sánchez en repetidas ocasiones le pidió al director de Educación el cambio de población argumentado lo siguiente: "Imploro se digne tomar en cuenta mis siguientes quejas en el Pueblo, donde presto mis humildes servicios, tropiezo con el lenguaje mexicano que yo no lo entiendo" ${ }^{26}$ Posteriormente, Sánchez sería destituida del pueblo de Tlalcuapan por las quejas de los mismos pobladores. ${ }^{27}$ Los casos anteriores enfatizan la importancia de contar con profesores hablantes de la lengua mexicana, al menos para estas poblaciones, sin importar los señalamientos que se hacían a nivel federal sobre el uso de las lenguas indígenas en la educación.

\footnotetext{
${ }^{24}$ Informe del inspector pedagógico de educación. AHET, Fondo: FRRO, Sección: IP, Año: 1921, Caja: 366, Exp: 22, Fs: 2, Chiautempan, Tlaxcala.

${ }^{25}$ Carta del agente municipal Manuel Rugerio al director de educación. AHET, Fondo: Histórico, Sección: SEP, Año: 1926, Caja: 145, Exp: 77, Chiautempan, Tlaxcala.

${ }^{26}$ Carta de Josefina Sánchez al director de educación. AHET, Fondo: Histórico, Sección: SEP, Año: 1922, Caja: 73, Exp: 10 y 12 , Chiautempan, Tlaxcala.

${ }^{27}$ Cartas con quejas de los padres de familia. AHET, Fondo: Histórico, Sección: SEP, Año: 1922, Caja:73, Exp: 10 y 12. Chiautempan, Tlaxcala.
} 


\section{Consideraciones finales}

En este trabajo se presentaron a una escala municipal prácticas y comportamientos que suceden en los ámbitos escolares y que permiten descubrir esa heterogeneidad en la historia de la educación mexicana. La intención de mirar lo que sucedía en un espacio como el pueblo me dio la oportunidad de conocer qué tanto se usaba una lengua indígena en la educación de los niños. El objetivo principal de este trabajo fue rescatar a algunos profesores que utilizaron la lengua mexicana de una forma natural y cotidiana en clases, justo cuando se desconocían y debatían las utilidades educativas y castellanizadoras de las lenguas vernáculas en el sistema educativo mexicano.

En Tlaxcala no existió reglamentación o ley que señalara el uso del mexicano, ni política educativa lingüística a nivel estatal. En estas escuelas la práctica bilingüe se llevó a cabo de una manera más informal. Utilizar el mexicano en clases le proporcionó al profesor mayor apoyo en el desarrollo de las mismas, sobre todo con niños pequeños monolingües que dependían del profesor para comprender sus lecciones y aprender español.

El uso oral del mexicano facilitó las clases y la aceptación con los padres de familia de los pueblos hablantes; fue base, al menos para estos pueblos, de su castellanización. Los padres de familia eran sujetos activos en torno a su espacio educativo local, por tanto, adaptaron o asimilaron a sus propias realidades la educación de sus hijos. El uso auxiliar de la lengua materna de los niños ocasionó que los conocimientos y aprendizajes fueran más eficaces, por ello los padres sabían de antemano, que un profesor no hablante de mexicano tendría más dificultades de enseñanza que uno bilingüe.

Los avances en los aprendizajes y en el conocimiento del español como segunda lengua eran muy avalados por los padres de familia, además del reconocimiento a que se hacían acreedores los profesores en los pueblos. La insistencia del uso oral del mexicano como auxiliar educativo facilitó la comunicación y la enseñanza en las escuelas de estos pueblos.

Así pues, este trabajo invita a mirar las escuelas de los pueblos hablantes de una lengua indígena como espacios para entender cómo se desarrollaba el rubro educativo, en contraparte a los grandes procesos educativos nacionales. Sostengo la hipótesis de que la lengua mexicana era algo común y cotidiano en estos poblados y no había preocupación en el sistema educativo estatal por su uso.

Reconstruir los procesos educativos locales puede ser provechoso para conocer con mayor detenimiento diversas temáticas como el uso de una lengua ajena al español, la vida de los profesores y su relación con los pueblos y padres de familia, así como la vida cotidiana en las escuelas rurales.

El uso del náhuatl y de otra lengua ajena al español, es un tema que todavía falta explorar en la historia de las escuelas rurales mexicanas, sus relevancia e importancia en la formación y 
educación de los niños indígenas mexicanos son importantes para la historia de la educación y de los pueblos indígenas de México.

\section{Fuentes}

Archivos

AHET Archivo Histórico del Estado de Tlaxcala, Tlaxcala, México. Fondos: Histórico y Revolución y Álvaro Obregón.

AGN Archivo General de la Nación, Archivo Histórico de la Secretaría de Educación Pública, Ciudad de México, México.

Fondo: Dirección General de Educación para Estados y Territorios

Fuentes Primarias Impresas

Secretaría de Fomento, Colonización e Industria (1906), División Territorial de la República Mexicana. Estado de Tlaxcala, formada. con los datos del censo verificado el 28 de octubre de 1900 a cargo del Doctor Antonio Peñafiel, Secretaría de Fomento, Colonización e Industria, Dirección General de Estadística, México.

(1918), Tercer Censo de Población, 1910. Estados Unidos Mexicanos, Secretaría de la Economía Nacional, Dirección General de Estadística, México.

Secretaría de la Economía Nacional (1943), Sexto Censo de Población, 1940. Estados Unidos Mexicanos. Tlaxcala, Secretaría de la Economía Nacional, Dirección General de Estadística, México. (Contiene cifras del Quinto Censo de Población, 1930)

Bibliografía

Acevedo, Ariadna (2011a), "La ignorada cuestión del idioma: educación en los pueblos indígenas de Puebla, México (1876-1930)" en Lourdes Alvarado y Rosalina Ríos (coords.), Grupos marginados de la educación (siglos XIx y XX), Universidad Nacional Autónoma de México, México, pp. 431-368.

Acevedo, Ariadna (2011b), "Muchas escuelas y poco alfabeto: la educación rural en el porfiriato, México, 1876-1910", en Alicia Civera, Juan Alfonseca y Carlos Escalante (coords.), Campesinos y escolares. la construcción de la escuela en el campo latinoamericano (siglos xIx y XX), Miguel Ángel Porrúa-El Colegio Mexiquense, México, pp. 73-106.

Acevedo, Ariadna (2012), "Las apariencias importan. Indumentaria e higiene personal como marcas de civilización y ciudadanía en la educación para campesinos e indígenas" en Ariadna Acevedo y Paula López Caballero (coords.), Ciudadanos inesperados: espacios de formación de la ciudadanía ayer y hoy, El Colegio de México-Centro de Investigación y de Estudios Avanzados del Instituto Politécnico Nacional, México, pp. 131-168.

Aguirre Beltrán, Guillermo (1973), Teoría y práctica de la educación indígena, Secretaría de Educación Pública, México.

Castillo Molina, Daniel Gibran (2017), "Una mirada a la gestión local, las prácticas docentes y el náhuat vistos desde las escuelas de los pueblos y barrios de Santa Ana Chiautempan (Tlaxcala), 1900-1930", tesis de maestría, Centro de Investigación y de Estudios Avanzados del Instituto Politécnico Nacional, Ciudad de México.

Cifuentes, Bárbara (1998), Letras sobre voces. Multilingüismo a través de la historia, Centro de Investigaciones y Estudios Superiores en Antropología Social, México. 
Cifuentes, Bárbara (2002), Lenguas para un pasado, huellas de una nación. Los estudios sobre lenguas indígenas de México en el siglo XIX. Plaza y Valdés editores-Consejo Nacional Para la Cultura y las Artes, México.

Coronado, Gabriela (1999), Porque hablar dos idiomas es como saber más: sistemas comunicativos bilingües ante el México plural, Centro de Investigaciones y Estudios Superiores en Antropología SocialConsejo Nacional de Ciencia y Tecnología, México.

Cuapio, Macrina (2010), Memoria histórica de San Francisco Tetlanohcan, Gobierno del Estado de Tlaxcala, México.

Escalante Fernández, Carlos (2006), "Educación, historia y región: la escala municipal”, en Lucía Martínez y Antonio Padilla (coords.), Miradas a la historia regional de la educación, Universidad Autónoma del Estado de Morelos-Miguel Ángel Porrúa-Consejo Nacional de Ciencia y Tecnología, México, pp. 77-92.

González y González, Luis (1997a), "Historia regional en sentido riguroso", Invitación a la microhistoria, Revista del Colegio Nacional de México, pp. 50-70.

González y González, Luis (1997b), Invitación a la microhistoria, El Colegio Nacional, México.

Heath, Shirley Brice (1970), La política del lenguaje en México. De la colonia a la nación, Instituto Nacional Indigenista, México.

Hill, Jane y Hill Kenneth (1999), Hablando mexicano: la dinámica de una lengua sincrética en el centro de México, Centro de Investigaciones y Estudios Superiores en Antropología Social, México.

Hobsbawm, Eric (1991), Naciones y nacionalismo desde 1780, Crítica-Crijalbo Mondodori, España.

Martín, Alicia y Carlos Escalante Fernández (2017), "Prácticas educativas, "El problema de la lengua" y usos indígenas de la lectura y la escritura en la historiografía de la educación del siglo xIx en México", Revista Mexicana de Investigación Educativa, núm. 73, vol. 22, pp. 347-366.

Messing, Jacqueline (2003), "Ideological Multiplicity in Discourse Language Shift and Bilingual Schooling in Tlaxcala, Mexico", Tesis de doctorado, Universidad de Arizona, Phoenix.

Messing, Jacqueline y Elsie Rockwell (2006), "Local language promoters and new discursive spaces: Mexicano in and out of schools in Tlaxcala", en Margarita Hidalgo (ed.), Mexican Indigenous Languages at the Dawn of the Twenty-First Century, Mouton Gruyter Berlin, Nueva York, pp. 249-280.

Plá, Dolores (2005), "Indígenas, mezclados y blancos. Según el censo general de habitantes de 1921", Historias, Revista del Instituto Nacional de Antropología e Historia, núm. 61, mayo-agosto, pp. 67-84.

Rockwell, Elsie (1994), "Schools of the Revolution: Enacting and Contesting State Forms in Tlaxcala, 1910-1930", en Joseph Gilbert y Daniel Nugent (eds.), Everyday Forms of State Formation: Revolution and the Negotiation of Rule in Modern Mexico, Duke University Press, Durham, North Caroline, pp. $170-208$.

Rockwell, Elsie (2004), "Entre la vida y los libros: prácticas de lectura en las escuelas de la Malintzi a principios del siglo xx”, en Carmen Castañeda, Luz Elene Galván y Lucía Martínez (coords.), Lectura y lectores en la historia de México, Centro de Investigaciones y Estudios Superiores en Antropología Social, México, pp. 327-357.

Rockwell, Elsie (2006a), "Apropiaciones indígenas de la escritura en tres dominios: religión, gobierno y escuela", Cultura Escrita y Sociedad, Revista internacional de historia social de la cultura escrita, núm. 3, octubre, pp. 161-218.

(2006b), "Historias contrastantes de la apropiación de la escritura en dos pueblos indios: los nahuas de Tlaxcala y los tzeltales de Chiapas", en María Bertely (coord.), Historias, saberes indígenas y nuevas etnicidades en la escuela, Centro de Investigaciones y Estudios Superiores en Antropología Social, México, pp. 35-68. 
(2007a), Hacer escuela, hacer estado. La educación posrevolucionaria vista desde Tlaxcala, El Colegio de Michoacán, Centro de Investigaciones y Estudios Superiores en Antropología Social-Centro de Investigación y de Estudios Avanzados del Instituto Politécnico Nacional, México.

(2007b), "Relaciones con la cultura escrita en una comunidad nahua a principios del siglo xx: temas recurrentes en los relatos orales", en Antonio Castillo (dir.) y Verónica Sierra (ed.), Senderos de ilusión. Lecturas populares en Europa y América Latina (Del siglo XVI a nuestros días), Trea ediciones, España, pp. 259-278.

y Oresta López (1992), "Entrevista al profesor rural Cluadio Hernández", Contla, Tlaxcala, 1 de abril de 1992, entrevista proporcionada por la primera autora.

Urquía, Manuel (1942), Estado de Tlaxcala. Breve examen de su situación económica y social, Gobierno del Estado de Tlaxcala, México.

Vázquez Santa Anna, Higinio (1928), Apuntes geográficos e históricos del estado de Tlaxcala, Gobierno del Estado de Tlaxcala, México.

Daniel Gibran Castillo Molina es asistente pedagógico del programa de Maestría en Educación en Biología para la Formación Ciudadana del Centro de Investigación y de Estudios Avanzados del Instituto Politécnico Nacional (Cinvestav) Unidad Monterrey, México. Es Maestro en Ciencias en la Especialidad en Investigaciones Educativas por el CINVESTAV. Sus líneas de Investigación son historia de las lenguas en la educación en México e historia del México posrevolucionario.

Recibido: 2 de junio de 2017

Aceptado: 24 de julio de 2017 